\title{
Lightweight Optical Constellation Modeling by Concatenating Artificial Neural Networks
}

\author{
D. Sequeira ${ }^{\left(1^{*}\right)}$, M. Ruiz(1), N. Costa(2), A. Napoli(3), J. Pedro(2), and L. Velasco(1) \\ (1) Universitat Politècnica de Catalunya (UPC), Barcelona, Spain ("diogo.goncalo.sequeira@upc.edu) \\ (2) Infinera Unipessoal Lda., Carnaxide, Portugal (3) Infinera, Munich, Germany
}

\begin{abstract}
A$ lightweight optical constellations modeling method based on concatenating ANNs is proposed. Statistical validation of the reproduced constellations is shown. The method accelerates data generation and facilitates detecting (un)intentioned misconfigurations, among others.
\end{abstract}

\section{Introduction}

In the recent years, Machine Learning (ML) algorithms have been extensively applied to optical communications to enhance their performance [1]. Applications range from identifying, predicting, and monitoring optical transmission parameters to mitigate different physical layer impairments, such as chromatic dispersion and non-linearities. Those applications require large data sets for training $\mathrm{ML}$ models; although such data should come from experimental setups, much research is being carried out using accurate heavy simulation environments (e.g., VPIphotonics) and analytical models (e.g., GNPy). Besides, some $\mathrm{ML}$ applications use an expected signal as a reference, e.g., to detect degradations ${ }^{[2]}$.

In this paper, we propose an accurate lightweight modeling of the physical layer impairments introduced by Reconfigurable Optical Add/Drop Multiplexers (ROADM) and optical links including intermediate Optical Amplifiers (OA) on the optical constellation; it is based on feedforward Artificial Neural Networks (ANN). The key advantage of our approach is that such ANNs can be concatenated to reproduce the impairments of an end-to-end lightpath, connecting one transmitter $(T x)$ and one receiver $(\mathrm{Rx})$.

\section{Methodology}

Fig. 1 illustrates the proposed concatenation model. A typical lightpath is represented in Fig. $1 \mathrm{a}$, connecting a Tx to a Rx. Between them, the signal may cross several ROADMs and optical links. ROADMs are modelled with two Wavelength Selective Switches (WSS), and every intermediate ROADM, except the last one before the Rx (drop), includes a booster OA that compensates for WSSs insertion losses. Typically, the insertion losses in the drop ROADM are compensated by digital signal processing (DSP) at the digital coherent Rx. The optical links consist of a pair of optical fibers followed by inline OAs set to compensate the losses of the fiber spans. We assume that the pre-OA at ROADM's input is a part of the link model, as shown in insets of Fig. 1a. The received optical constellation contains information about the devices that the optical signal has crossed and it can be used, for example, to detect degradation.

The concatenation model abstracting the lightpath is presented in Fig. 1b. In this case, the ROADMs and optical links in Fig. 1a are modeled as ANNs. The resulting ANNs are concatenated in the same order as they appear in the route of the lightpath to create the end-to-end concatenation model, therefore reproducing the complete lightpath. To achieve an accurate concatenation model, the ANN models use input features related to the optical constellation and output the resulting features after crossing the modeled optical system, i.e., a ROADM or a fiber link.

Two operations are key to implement the proposed approach: i) the generator modelling the $\mathrm{Tx}$ and ii) the constellation reconstruction at the end of the chain. The generator uses an input bit sequence to generate optical signal samples following a Tx model, which can be implemented using analytical equations, simulation, or $\mathrm{ML}$ models. After the Tx model computes the initial optical constellation, a feature extraction block computes the input features of the concatenation model. Features include mean and variance of selected constellation points (detailed in the next section). The output of the concatenation model is used by the constellation reconstruction module to generate the optical constellation from the received features of the selected constellation points after propagation through the concatenation model.

The proposed approach can be used as a lightweight optical system simulator. Note that the constellation at intermediate points in the lightpath can be also obtained by just reconstructing the constellation from the desired features. It is worth noting that the time to generate the resulting optical constellation is noticeably short as it entails propagating values through a set of ANNs, i.e., only a very limited number of simple calculations is required ${ }^{[3]}$.

\section{Feature Extraction and Modeling}

We model the constellation points as bivariate Gaussian distributions. Therefore, constellation 


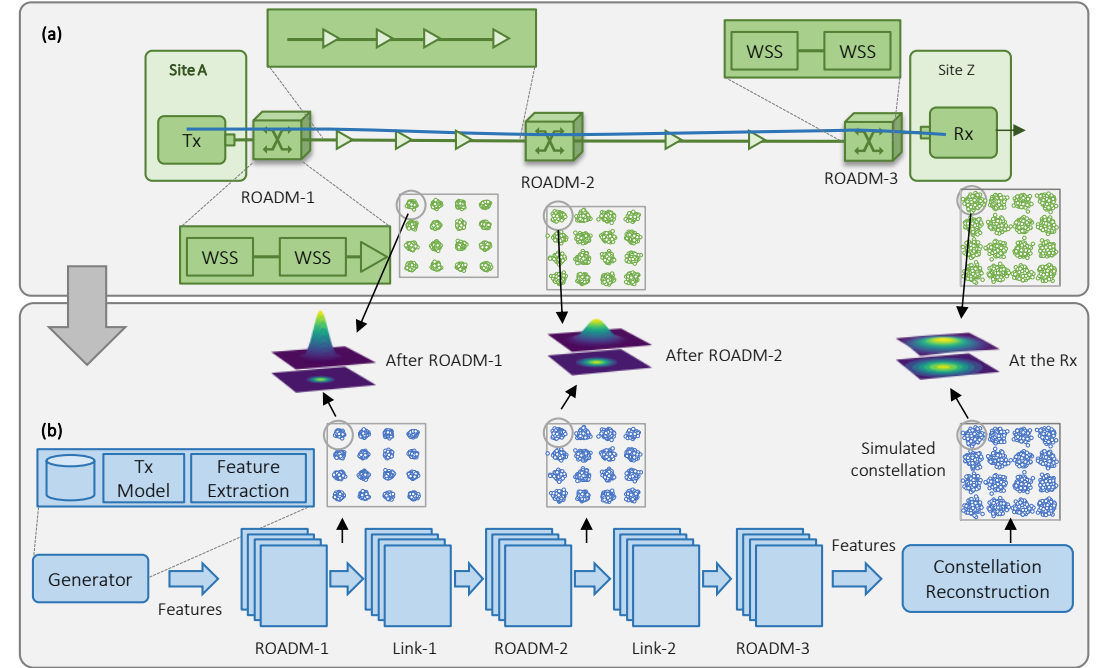

Fig. 1: Concatenation modeling

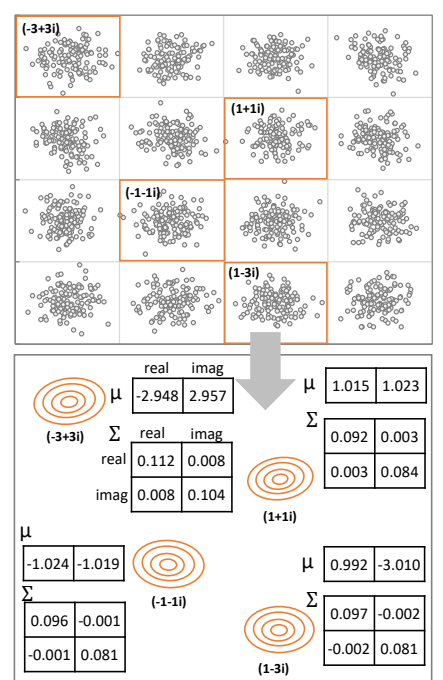

Fig. 2: Feature computation points features are computed by applying Gaussian Mixture Models (GMM) ${ }^{[4]}$, as shown in Fig. 2. This approach characterizes every constellation point $p$ with a two-dimensional vector $\mu_{p}$ representing the mean in the constellation and with a $2 \times 2$ matrix $\Sigma_{p}$, which captures the variance (diagonal of $\Sigma_{p}$, hereafter referred to as $\sigma_{p}$ ) and the covariance that the symbols belonging to the constellation point $p$ experienced around the mean position. In order to find accurate ANN models for a wide range of scenarios (including those not considered during training), a common practice is to select a reduced number of model inputs ${ }^{[5]}$. Hence, we limit the number of constellation points to the minimum providing enough information to capture the overall constellation behavior; specifically for 16QAM, we selected two outer ($3+3 i, 1-3 i)$ and two inner $(1+1 i$ and $-1-1 i)$ constellation points (see Fig. 2).

We consider that both the models for the optical fiber links and ROADMs follow the same architecture characterized by: I) 24 input neurons (6 features per constellation point); ii) two hidden layers, each one with 12 neurons and hyperbolic tangent activation function [3]; and iii) one output layer with 24 neurons that estimates the output constellation's features. For example, when the lightpath crosses two ROADMs and one link, the proposed approach entails concatenating 3 ANN models, which results into 216 neurons, from which 72 are hidden neurons implementing an activation function.

Constellation reconstruction is done by firstly computing all constellation point features by means of linear combinations of the propagated features (to be fitted during ANN training phase). Then, the simulated constellations can be obtained by randomly sampling bivariate Gaussian distributions for all constellation points.

\section{Illustrative Results}

To evaluate the performance of the proposed approach, a MATLAB-based digital coherent system simulator has been implemented. We assume a 11 channel WDM system, where all channels are configured with 16QAM@64GBd and $75 \mathrm{GHz}$ channels spacing. At the transmitter side, pseudo-random binary sequences of $2^{15}$ bits are modulated and shaped by a root-raised cosine filter with a roll-off factor of 0.06 . Next, an optical multiplexer aggregates the individual signals and creates the WDM signal to be propagated through the lightpath. A per channel launch power of $-1 \mathrm{dBm}$ is assumed. The optical fiber spans are composed of standard single mode fiber modelled by an attenuation factor of $0.21 \mathrm{~dB} / \mathrm{km}$ and a chromatic dispersion parameter of $16.8 \mathrm{ps} / \mathrm{nm} / \mathrm{km}$. The pulse propagation is modeled by solving the nonlinear Schrödinger equation using the split-step Fourier method with a propagation step size of $100 \mathrm{~m}$. Erbium doped fiber amplifiers with noise figure of $4.5 \mathrm{~dB}$ are considered. The WSSs inside the ROADMs are based on commercially available ones and modelled as described in [6]. Finally, DSP blocks capable to perform ideal chromatic dispersion compensation and carrier phase recovery are considered in the $\mathrm{Rx}$.

The simulator was used to generate the datasets needed for training, testing, and validating the models. Four different optical link configurations in terms of total length and number of spans are considered: $100-\mathrm{km}$ (2×50-km spans), $240-\mathrm{km}$ (4×60-km spans), 400-km (5×80-km spans), and $560-\mathrm{km}(7 \times 80-\mathrm{km}$ spans). For each one, up to 4 hops were considered leading to a maximum total lightpath length of 400, 960, 1600, $2240 \mathrm{~km}$, respectively. To train the models, 30 signal samples with around 8200 symbols each were generated for each link and ROADM configuration. 


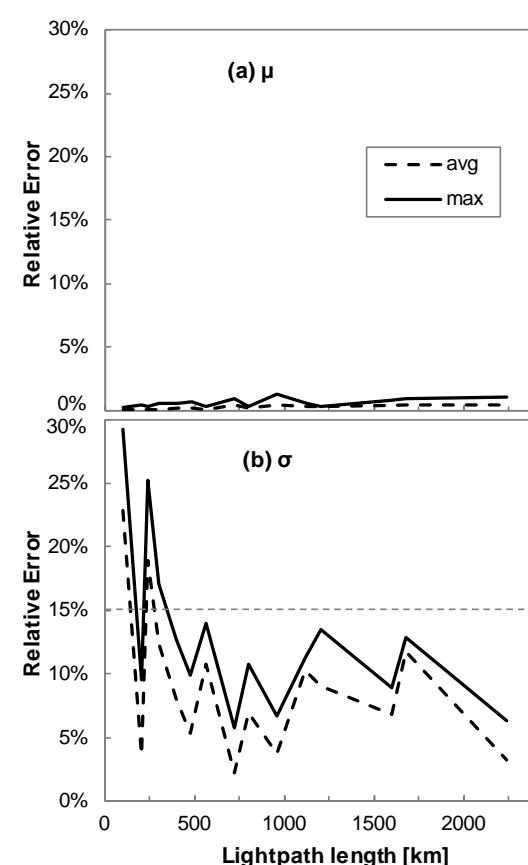

Fig. 3: Relative model error vs distance

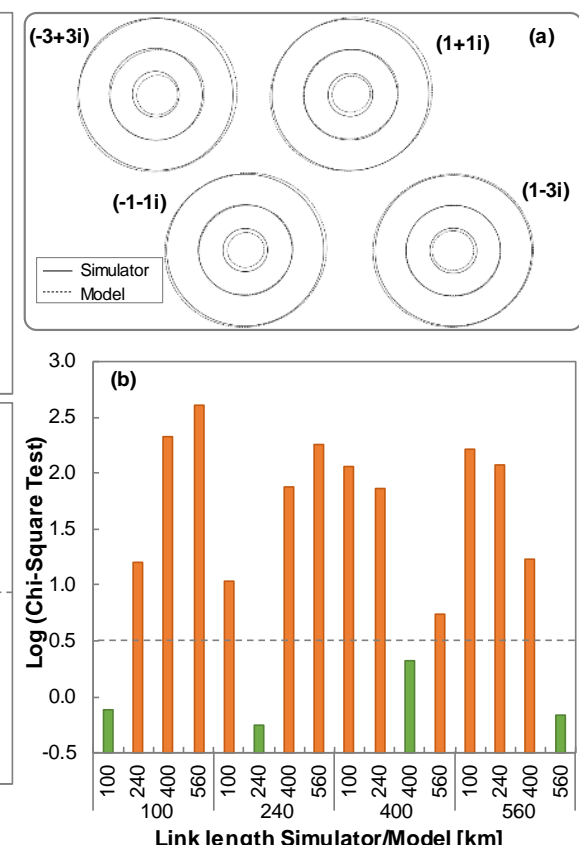

Fig. 4: Comparison simulator/model

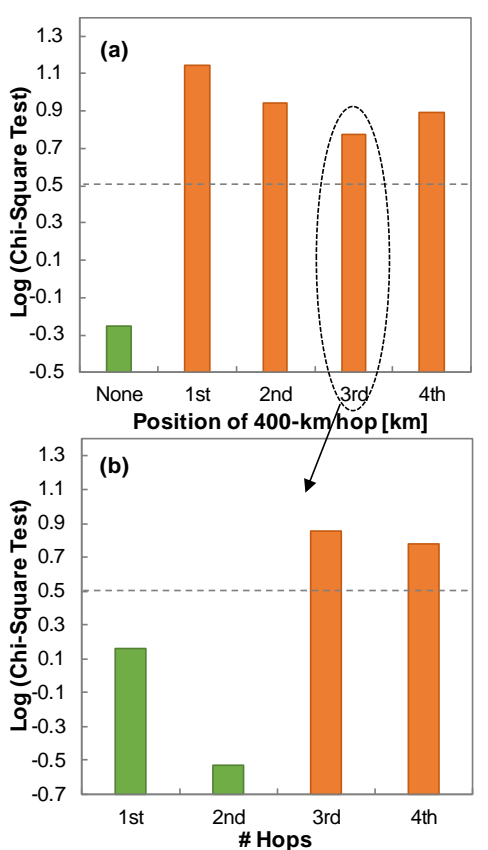

Fig. 5: Fine detection and localization
Each ANN was trained during 5,000 epochs; the overall absolute and relative errors for all link configurations, lightpath lengths, and selected constellation points are shown in Fig. 3. Since covariance terms in $\Sigma$ are nearly 0 , we focus on the average and maximum errors of features $\mu$ (Fig. 3a) and $\sigma$ (Fig. 3b). While the $\mu$ prediction errors are negligible independently of the link length ( $\max$ error $<2 \%$ ), the $\sigma$ max error is around $30 \%$ for low $\sigma$ values. However, as soon path length increases, maximum error decreases below $15 \%$, which is, in general, a good enough performance to validate the models. For illustrative purposes, Fig. 4a plots the Gaussian distributions for the selected constellation points, obtained with the concatenation model and simulator for a 1,600-km lightpath (4 hops of $400 \mathrm{~km}$ ). Strong similarities between both cases are evident.

The constellation reconstruction performance is now analyzed. To this aim, the features obtained with the model (including reconstruction) are compared to those extracted from the simulator. The comparison between model-based and simulator-based features was statistically carried out by means of the Chi-Square test to evaluate whether the two independent data sets are similar or conversely, significantly different ${ }^{[7]}$. We first compared the case when the simulation and model were configured with the same lightpath length and link configuration; for all the combinations, the hypothesis of equality was largely accepted (with decision error < 0.01\%). Second, we compared different configurations of 4-hop lightpaths to check if the value of the ChiSquare test statistic serves as indicator of misleading configuration of the model. Fig. 4b reports these results, showing that a threshold of 0.5 allows to clearly distinguish all cases when simulation and model were configured differently (orange) from cases with the same setup (green). Additionally, the impact of considering just slightly different scenarios in the simulation and concatenation model was tested. Specifically, a 4-hop lightpath with 240-km links was configured in the simulation, whereas model was configured with the same number of hops and link configuration except for only one of the hops, where a $400-\mathrm{km}$ link was selected. The four different positions in the path for the $400-\mathrm{km}$ link were evaluated; Fig. 5a shows that all cases stayed above the 0.5 threshold, which implies that the small difference was correctly detected. Note that localization of the longer link can be done by performing the test at intermediate links. Finally, Fig. 5b shows the result of applying this intermediate analysis when the $400-\mathrm{km}$ is in the third link. As it can be observed, the link is localized as the Chi-Square test value exceeds the selected threshold when evaluating the features right after the third link.

To conclude, we have proposed and assessed a lightweight method to generate optical constellations by concatenating ANN models. Statistical evidence of similarity between simulated and modeled constellations as well as a solid and robust detection method to recognize differences have been shown.

\section{Acknowledgements}

This work has been partially supported by the EC through the MSC REAL-NET project (G.A. 813144), by the AEI/FEDER through the TWINS project (TEC2017-90097-R), and by the ICREA institution. 


\section{References}

[1] D. Rafique and L. Velasco, "Machine Learning for Optical Network Automation: Overview, Architecture and Applications," (Invited Tutorial) IEEE/OSA Journal of Optical Communications and Networking (JOCN), vol. 10, pp. D126-D143, 2018.

[2] B. Shariati et al., "Learning from the Optical Spectrum: Failure Detection and Identification [Invited]," IEEE/OSA Journal of Lightwave Technology (JLT), vol. 37, pp. 433-440, 2019.

[3] C. Aggarwal, Neural Networks and Deep Learning: A Textbook, $1^{\text {st }}$ Ed., Springer International Publishing, 2018.

[4] N. Bouguila and W. Fan, Mixture Models and Applications, $1^{\text {st }}$ Ed., Springer International Publishing, 2020.

[5] V. Bolón-Canedo, N. Sánchez-Maroño, and A. AlonsoBetanzos, Feature Selection for High-Dimensional Data, $1^{\text {st }}$ Ed., Springer, 2015.

[6] T. Rahman et al., "On the Mitigation of Optical Filtering Penalties Originating from ROADM Cascade," IEEE Photonics Techn. Letters, vol. 26, pp. 154-157, 2014.

[7] R. Kuehl, Design of Experiments: Statistical Principles of Research Design and Analysis, $2^{\text {nd }}$ Ed., Duxbury Press, 1999. 